\title{
12 INTERVENÇÃO COMUNITÁRIA \& RECOVERY: IMPACTO NA QUALIDADE DE VIDA, SUPORTE SOCIAL E SATISFAÇÃO DE NECESSIDADES DA PESSOA COM DOENÇA MENTAL
}

\author{
| Ermelinda Macedo $;$ Filomena Gomes²; Analisa Candeias³ Carla Azevedo4; Sílvia Peixoto5; Bárbara Pires ${ }^{6}$ |
}

\section{RESUMO}

CONTEXTO: O recovery é entendido como um processo subjetivo que pressupõe as dimensões clínica e pessoal. Os programas de intervenção comunitária na promoção do recovery devem basear-se em aspetos importantes como a qualidade de vida, suporte social e necessidades.

OBJETIVOS: Avaliar o impacto de um programa de intervenção individualizado na qualidade de vida, suporte social e satisfação das necessidades, em pessoas com doença mental.

MÉTODOS: Estudo quase-experimental com desenho antes-após de grupo único, com três momentos de avaliação com 16 sessões implementadas durante 4 meses. A amostra é constituída por 25 pessoas com doença mental selecionadas a partir da alta clínica de um hospital psiquiátrico da região Norte de Portugal. Utilizaram-se como instrumentos: Questionário de Avaliação Sociodemográfica e Clínica; Instrumento de Avaliação de Necessidades, construídos pelos investigadores; EQ-5D e; Escala de Satisfação com o Suporte Social. A Investigação foi aprovada pela Comissão de Ética da instituição onde foram recolhidos os dados. Foi utilizado o SPSS, versão 25.0, com recurso a medidas descritivas e testes de diferenças de médias não paramétricos - Friedman e de Wilcoxon - com um valor de significância de p<.05.

RESULTADOS: Verificou-se uma evolução positiva relativamente às variáveis avaliadas, com diferenças estatisticamente significativas ao longo dos três momentos de avaliação para: qualidade de vida - EQ-5D; Dimensão Doença do IAN, e Dimensão Ambiente.

CONCLUSÕES: Intervir no recovery da pessoa com doença mental é uma intervenção morosa e exigente, mas com resultados positivos desde que a intervenção seja direcionada às necessidades das pessoas.

\section{PALAVRAS-CHAVE: Saúde mental; Recuperação; Qualidade de vida; Suporte social}

\section{RESUMEN}

"Intervención comunitaria \& recuperación: Impacto en la calidad de vida, soporte social y satisfación de las necesidades de la persona con enfermedad mental"

CONTEXTO: La recuperación es un proceso subjetivo que presupone las dimensiones clínica y personal. Los programas de intervención comunitaria en la recuperación deben basarse en la calidad de vida, el apoyo social y las necesidades.

OBJETIVOS: Evaluar el impacto de un programa de intervención individualizado en la calidad de vida, apoyo social y satisfacción de las necesidades, en personas con enfermedad mental.

METODOLOGÍA: Estudio casi experimental con diseño antes-después de grupo único, con tres momentos de evaluación con 16 sesiones implementadas durante 4 meses. La muestra se compone de 25 personas con enfermedad mental seleccionados a partir del alta clínica de un hospital psiquiátrico en el norte de Portugal. Se utilizaron como instrumentos un cuestionario sociodemográfico y clínico, el Instrumento de Evaluación de Necesidades, construidos por los investigadores, el EQ-5D y la Escala de Satisfacción con el soporte social. La investigación fue aprobada por la Comisión de Ética de la institución donde se recogieron los datos. Se utilizó el SPSS, versión 25.0, con medidas descriptivas y pruebas de diferencias de promedios no paramétricos - Friedman y Wilcoxon - con un valor de significancia de $\mathrm{p}<.05$.

RESULTADOS: Se observó una evolución positiva con respecto a las variables evaluadas, con diferencias estadísticamente significativas a lo largo de los tres momentos de evaluación para: calidad de vida - EQ-5D; Dimensión de la enfermedad del IAN, y la dimensión ambiental.

CONCLUSIONES: Intervenir en la recuperación de la persona con enfermedad mental es algo moroso y exigente, pero con resultados positivos desde que la intervención se dirija a las necesidades de las personas.

DESCRIPTORES: Salud mental; Recuperación; Calidad de vida; Apoyo social

\section{ABSTRACT \\ "Community intervention \& recovery: Impact on quality of life, social support and needs' satisfaction of the person with mental illness"}

BACKGROUND: Recovery is understood as a subjective process that presupposes clinical and personal dimensions. Community intervention programs in the promotion of recovery should be based on important aspects such as their needs, quality of life and social support.

AIM: To evaluate the impact of an individualized intervention program on quality of life, social support and needs satisfaction in people with mental illness. METHODS: Quasi-experimental study with before-after design of single group, with three evaluation moments with 16 sessions implemented during 4 months. The sample consists of 25 people with mental illness selected from the high clinic of a psychiatric hospital in the Northern region of Portugal. The sociodemographic and clinical evaluation questionnaire, the Needs Assessment Instrument (NAI)., constructed by the researchers, the EQ-5D and the Social Support Satisfaction Scale were used as instruments. The investigation was approved by the Ethics Committee of the institution where the data were collected. SPSS, version 25.0 was used, using descriptive measures and tests of non-parametric mean differences - Friedman and Wilcoxon - with a significance level of $\mathrm{p}<.05$.

RESULTS: There was a positive evolution regarding the evaluated variables, with statistically significant differences during the three evaluation moments for: quality of life - EQ-5D; Dimension Disease of the NAI, and Environment Dimension.

CONCLUSIONS: Intervening in the recovery of the person with mental illness is a time-consuming and demanding intervention, but with positive results if the intervention is directed to the needs of the people.

\section{KEYWORDS: Mental health; Recovery; Quality of life; Social} support

Submetido em 30-12-2018

Aceite em 01-03-2019

1 Doutora em Psicologia; Professora Adjunta na Universidade do Minho, Escola Superior de Enfermagem, Campus de Gualtar, Braga, Portugal, emacedo@ese.uminho.pt 2 Doutora em Enfermagem; Professora Coordenadora na Universidade do Minho, Escola Superior de Enfermagem, Braga, Portugal, fgomes@ese.uminho.pt 3 Mestre em Enfermagem de Saúde Mental e Psiquátrica; Mestre em Gestão; Professora Adjunta na Universidade do Minho, Braga, Portugal, acandeias@ese.uminho.pt 4 Mestre em Enfermagem; Enfermeira nas Irmãs Hospitaleiras, Casa de Saúde do Bom Jesus, Braga, Portugal, carla.bcl@hotmail.com 5 Mestre em Enfermagem; Enfermeira nas Irmãs Hospitaleiras, Casa de Saúde do Bom Jesus, Braga, Portugal, silvia_llp@hotmail.com

6 Mestranda em Enfermagem na Universidade do Minho, Escola Superior de Enfermagem, Braga, Portugal, barbara.8.pires@gmail.com

Citação: Macedo, E., Gomes, F., Candeias, A., Azevedo, C., Peixoto, S., \& Pires, B. (2020). Intervenção comunitária \& recovery: Impacto na qualidade de vida, suporte social e satisfação de necessidades da pessoa com doença mental. Revista Portuguesa de Enfermagem de Saúde Mental (Spe7), 81-87. 


\section{INTRODUÇÃO}

O recovery é entendido como um processo subjetivo que pressupõe as dimensões clínica e pessoal. Assim, e inseridas na primeira, encontram-se as dinâmicas que compreendem a recuperação das funções sociais, bem como o tratamento dos sintomas relacionados com a doença. Na segunda dimensão, estão patentes aspetos mais subjetivos e individuais, como a manifestação da experiência vivida com a doença mental, com ênfase na mudança de atitudes, de valores, de sentimentos, de capacidades, no delineamento de objetivos pessoais, e na mudança e adaptação a novos papéis, ou aos já existentes (Slade, 2013).

O conceito de recovery compreende, deste modo, um processo subjetivo e vasto. Este facto indica-nos que o recovery se deve centrar numa avaliação individual, fornecendo dados que permitam uma intervenção direcionada às necessidades/potencialidades de cada pessoa. A avaliação individual inicial neste processo visa compreender quais os aspetos a incluir num programa de intervenção posterior, e que este seja orientado, em conjunto com a pessoa, de uma forma concreta e objetiva - embora tendo em conta algumas variáveis que o poderão condicionar, como por exemplo a qualidade de vida (QdV), o suporte social e as necessidades da própria pessoa.

Em relação à $\mathrm{QdV}$, esta tem sido encarada como um resultado em saúde, tanto no que diz respeito à mortalidade como à morbilidade. A Organização Mundial de Saúde definiu QdV como "a percepção do indivíduo sobre a sua posição na vida, dentro do contexto dos sistemas de cultura e valores nos quais está inserido e em relação aos seus objectivos, expectativas, padrões e preocupações" (WHOQOL Group, 1996, p. 354). A QdV tem tido uma atenção especial por parte dos investigadores, embora esta atenção seja mais recente na saúde mental e psiquiatria. Apesar disso, já se observam alguns trabalhos envolvendo população diversa, como por exemplo, pessoas com diagnósticos psiquiátricos como depressão major (Gameiro, Carona, Silva e Canavarro., 2010, Macedo, 2013); doença bipolar (Figueira, Leitão e Gameiro, 2010; Macedo, 2013); a esquizofrenia (Vaz-Serra, 2010) ou; sintomatologia depressiva (Gameiro et al., 2008; Macedo, 2013).

Ao longo da vida, o suporte social é construído através das relações estabelecidas com os membros da família, colegas de trabalho e pessoas da comunidade e, em caso de presença de necessidades especiais, de um membro resultante de profissões de ajuda (Cobb, 1976).
Estudos efetuados para analisar o efeito do suporte social em pessoas com doença mental revelam que este tem um efeito positivo na adaptação destas pessoas, reduzindo também os sintomas psiquiátricos e o número de hospitalizações (Huang, Sousa, Tsai, \& Hwang, 2008).

Um suporte social mais baixo é uma razão importante para a diminuição da satisfação com a vida e igualmente uma razão para o aumento de sintomas depressivos entre a população mais idosa (Newson \& Schulz, 1996). Em pessoas com doença mental, o contacto com a rede social e maiores níveis de suporte social foram associados à utilização de menos serviços dentro do âmbito da psiquiatria (Maulik, Eaton, \& Bradshaw, 2009).

As necessidades das pessoas com doença mental são abrangentes e incluem a QdV, relações interpessoais, domínio social, autoeficácia, definição e realização de objetivos e a estabilização de sintomas psiquiátricos (Gelkopf et al, 2016). Ainda no que diz respeito ao processo de recovery, existem programas orientados para a maximização dos resultados e centrados no modelo comunitário, que visam a integração da pessoa com doença mental nos contextos comunitários. Estes programas devem basear-se em aspetos como a orientação e envolvimento da pessoa no processo, a autodeterminação e a perceção de potencialidades por parte dos profissionais de saúde que estão a acompanhar o processo (Anastácio, \& Furtado, 2012), dados que são fornecidos através de uma avaliação que anteceda o programa de intervenção que deve incluir dimensões da vida que vão para além daquelas que o recovery clínico pressupõe. Tendo em conta os pressupostos do processo de recovery da pessoa com doença mental, o objetivo deste trabalho foi avaliar o impacto de um programa de intervenção individualizado na qualidade de vida, suporte social e satisfação das necessidades nesta população.

\section{MÉTODOS}

Trata-se de um estudo quase-experimental com desenho antes-após, de grupo único (Fortin, 2009), inserindo-se numa investigação mais alargada, cujo objeto de estudo é o recovery da pessoa com doença mental. $\mathrm{O}$ estudo foi realizado contando com uma equipa multidisciplinar e insere-se na terceira fase da investigação, que diz respeito à seleção da amostra e implementação de um programa de intervenção individual domiciliário. 
O programa de intervenção englobou 16 sessões (1 sessão por semana, durante 4 meses), realizando-se três momentos de avaliação: avaliação inicial (M0), efetuada aquando a alta hospitalar e antecedendo a intervenção; avaliação intermédia (M1), efetuada na $8^{\text {a }}$ sessão, coincidindo com o $2^{\circ}$ mês após a alta hospitalar e avaliação final (M2), efetuada após a implementação das 16 sessões do programa de intervenção, coincidindo com o $4^{\circ}$ mês após a alta hospitalar. O programa de intervenção desenhou-se a partir do M0, isto é, através dos resultados que se obtiveram com a aplicação dos instrumentos de avaliação, e foi-se aferindo de acordo com a evolução dos participantes nas dimensões inicialmente identificadas como as mais vulneráveis. Esta maior vulnerabilidade, globalmente, incidiu sobre as dimensões: relações sociais; doença; psicológica; serviços de saúde e profissionais e ambiente e; económica, sendo delineados objetivos para cada participante em cada sessão e, consequentemente, aferidas as intervenções em função dos resultados obtidos.

Foram aplicados os mesmos instrumentos de recolha de dados nos diferentes momentos de avaliação, nomeadamente: Questionário de Dados Sociodemográficos e Clínicos (construído pela equipa de investigação).

Para avaliar a QdV aplicou-se o EQ-5D, instrumento genérico multidimensional. Da aplicação deste instrumento, é gerado um Índice do Estado de Saúde (IES), com valores entre $-0,59$ a 1 , sendo que os valores negativos correspondem a estados de saúde piores que a morte, o 0 corresponde ao estado de saúde igual à morte, e o 1 corresponde à saúde perfeita. Para além deste índice, existe uma Escala Visual Analógica (EQVAS), em que a pessoa classifica o seu Estado de Saúde no Momento (ESM), com scores entre 0 (pior saúde imaginável) e 100 (a melhor saúde imaginável). O EQ5D apresenta ainda uma questão que pretende comparar o estado de saúde atual com o estado de saúde de há um ano atrás (Ferreira, Ferreira, Pereira, 2013). A avaliação da satisfação do suporte social foi realizada com recurso à Escala de Satisfação com o Suporte Social (ESSS) (Pais-Ribeiro, 2011).

Esta Escala é constituída por 15 itens, e score total final entre 15 e 75. Foi também utilizado um Instrumento de Avaliação de Necessidades (IAN), igualmente construído pela equipa de investigação com base num trabalho prévio de revisão da literatura e análise de entrevistas a peritos na área da saúde mental com experiência no âmbito do recovery, com 44 itens distribuídos em seis dimensões:
Dimensão Relações Sociais (RS), Dimensão Doença (D), Dimensão Económica (E), Dimensão Serviços de Saúde e Profissionais (SSP), Dimensão Psicológica (P) e Dimensão Ambiente (A).

Os casos foram selecionados no momento da alta hospitalar, a partir de uma técnica de amostragem não aleatória (conveniência) $(\mathrm{N}=25)$. Para este estudo foram incluídos todos os indivíduos que participaram nas 16 sessões do programa de intervenção ao longo dos 4 meses de implementação, tendo sido alvo de avaliação nos 3 momentos de avaliação do estudo. Foram eleitos como critérios de inclusão: pessoas com doença mental em internamento, com alta prevista para domicílio, com idade igual ou superior a 18 anos e a residirem na região da instituição onde os participantes foram sujeitos a internamento. Excluíram-se os casos com diagnósticos de demência, problemas de adição e debilidade intelectual.

Para o tratamento e análise dos dados foi utilizado o Statistical Package for the Social Sciences, versão 25.0, com recurso a medidas descritivas e medidas inferenciais não-paraméticas - testes de Friedman e Wilcoxon - testes estes utilizados para averiguar se existem diferenças de médias nos valores das variáveis avaliadas, respetivamente, nos 3 momentos de avaliação do estudo $(\mathrm{M} 0 \rightarrow \mathrm{M} 1 \rightarrow \mathrm{M} 2)$ e entre o $1^{\circ}$ e $3^{\circ}$ momento $(\mathrm{M} 0 \rightarrow \mathrm{M} 2)$, considerando um valor de significância de $\mathrm{p}<.05$ (Pestana e Gageiro, 2008).

O estudo foi avaliado, aprovado e financiado pela FCT - Fundação para a Ciência e Tecnologia, e obteve aprovação da Comissão de Ética da instituição onde foi selecionada a amostra. Todos os procedimentos éticos inerentes à investigação foram explicados a todos os participantes: os objetivos, o desenho e os procedimentos do estudo. Os participantes assinaram o consentimento informado livre e esclarecido, sendo garantida a confidencialidade dos dados, bem como o anonimato.

\section{RESULTADOS}

No que diz respeito à caraterização sociodemográfica da amostra, a totalidade é do sexo feminino, a média de idade é de 49,4 anos ( $\mathrm{DP}= \pm 12,08)$; $56 \%$ são casadas e $28 \%$ concluiu o $3^{\circ}$ ciclo, sendo que, $36 \%$ encontramse empregadas e $32 \%$ encontram-se reformadas. $76 \%$ pertence a uma família nuclear. $\mathrm{O}$ diagnóstico médico mais frequente pertence às perturbações do humor (44\%). 
Tabela 1 -Caraterização Sociodemográfica e Clínica dos Participantes

\begin{tabular}{|c|c|c|c|}
\hline \multicolumn{2}{|c|}{ Caraterização Sociodemográfica e Clínica } & \multirow{2}{*}{$\frac{\mathrm{No}^{\circ}}{0}$} & \multirow{2}{*}{$\begin{array}{c}\% \\
0,0\end{array}$} \\
\hline Sexo & Masculino & & \\
\hline & Feminino & 25 & 100,0 \\
\hline \multirow[t]{4}{*}{ Idade } & Média & \multicolumn{2}{|c|}{49,4} \\
\hline & Máximo & \multicolumn{2}{|c|}{69} \\
\hline & Mínimo & \multicolumn{2}{|c|}{22} \\
\hline & Desvio Padrão & \multicolumn{2}{|c|}{12,08} \\
\hline \multirow[t]{4}{*}{ Estado civil } & Casado/união de facto & 14 & 56,0 \\
\hline & Solteiro & 7 & 28,0 \\
\hline & Viúvo & 2 & 8,0 \\
\hline & Divorciado/separado & 2 & 8,0 \\
\hline \multirow{6}{*}{$\begin{array}{l}\text { Habilitações } \\
\text { literárias }\end{array}$} & Analfabeto & 0 & 0 \\
\hline & $1 .^{\circ}$ ciclo (4 anos) & 6 & 24,0 \\
\hline & $\begin{array}{l}\text { Ensino básico }-2 .^{\circ} \text { ciclo }(6 \\
\text { anos) }\end{array}$ & 2 & 16,0 \\
\hline & $\begin{array}{l}\text { Ensino básico }-3 .^{\circ} \text { ciclo }(9 \\
\text { anos) }\end{array}$ & 7 & 28,0 \\
\hline & $\begin{array}{l}\text { Ensino secundário (11 ou } 12 \\
\text { anos) }\end{array}$ & 5 & 20,0 \\
\hline & Ensino superior & 3 & 12,0 \\
\hline \multirow{4}{*}{$\begin{array}{l}\text { Situação } \\
\text { profissional }\end{array}$} & Estudante & 1 & 4,0 \\
\hline & Empregado(a) & 9 & 36,0 \\
\hline & Reformado(a) & 8 & 32,0 \\
\hline & Desempregado(a) & 7 & 28,0 \\
\hline \multirow{2}{*}{$\begin{array}{l}\text { Tipo de } \\
\text { família }\end{array}$} & Isolado & 6 & 24,0 \\
\hline & Família nuclear & 19 & 76,0 \\
\hline \multirow{3}{*}{$\begin{array}{l}\text { Diagnóstico } \\
\text { médico }\end{array}$} & Perturbação psicótica & 9 & 36,0 \\
\hline & Perturbação do humor & 11 & 44,0 \\
\hline & $\begin{array}{l}\text { Perturbação da personali- } \\
\text { dade }\end{array}$ & 5 & 20,0 \\
\hline
\end{tabular}

Quando se aplicam as medidas descritivas, nos resultados obtidos nos três momentos de avaliação, verificouse que todas as variáveis recorrentes dos instrumentos de avaliação sofreram, sem exceção, uma evolução positiva (Tabela 2). Atendendo aos sores possíveis do EQ-5D (-0,59 a 1) que a presentam scores favoráveis nos três momentos de avaliação, ou seja, mais próximos de 1. Relativamente ao ESSS, com scores entre 15 a 75 e não tendo a escala ponto de corte, parece que os sujeitos apresentam valores satisfatórios também nos três momentos. A qualidade de vida apresenta, contudo, uma evolução mais favorável. Em relação ao IAN, com pontuações entre 0 e 100 , verifica-se que a Dimensão económica é a dimensão mais fragilizada, seguida da Dimensão SSP e da dimensão ambiente.
Tabela 2 -Análises descritivas dos três momentos de avaliação relativas à QdV, Suporte Social e Satisfação das Necessidades, $(\mathrm{N}=25)$

\begin{tabular}{|l|l|l|l|}
\hline & \multicolumn{3}{|c|}{ Momentos de Avaliação } \\
\hline & \multicolumn{1}{|c|}{$\begin{array}{c}\text { M0 } \\
(\text { Média } \pm \text { DP })\end{array}$} & $\begin{array}{c}\text { M1 } \\
(\text { Média } \pm \text { DP })\end{array}$ & $\begin{array}{c}\text { M2 } \\
(\text { Média } \pm D P)\end{array}$ \\
\hline EQ-5D & $0,69 \pm 0,32$ & $0,84 \pm 0,12$ & $0,93 \pm 0,09$ \\
\hline ESSS & $47 \pm 11,85$ & $47,16 \pm 9,03$ & $50,80 \pm 10,71$ \\
\hline IAN & & & \\
\hline Dimensão RS & $16,56 \pm 3,78$ & $16,68 \pm 3,35$ & $17,76 \pm 2,73$ \\
\hline Dimensão D & $43,64 \pm 9,95$ & $45,8 \pm 5,0$ & $51,08 \pm 5,95$ \\
\hline Dimensão E & $12,28 \pm 3,74$ & $12,72 \pm 2,75$ & $13,00 \pm 1,87$ \\
\hline Dimensão SSP & $14,72 \pm 3,35$ & $15,96 \pm 3,0$ & $16,52 \pm 2,29$ \\
\hline Dimensão P & $41,68 \pm 10,24$ & $41,76 \pm 8,31$ & $44,12 \pm 6,09$ \\
\hline Dimensão A & $19,44 \pm 4,19$ & $20,12 \pm 3,22$ & $21,68 \pm 2,27$ \\
\hline
\end{tabular}

Através do Teste de Friedman (Tabela 3) foi possível verificar diferenças estatisticamente significativas ao longo dos três momentos de avaliação na a QdV - EQ-5D ( $\mathrm{p}<.001)$; na Dimensão Doença $(\mathrm{p}<.001)$ e; na Dimensão Ambiente $(\mathrm{p}=.001)$ do IAN. O Teste de Wilcoxon permite-nos verificar se existe alguma alteração posicional em termos dos valores médios obtidos nos momentos de avaliação e, para este estudo, na maioria, esta alteração ocorreu. Assim, comparando os momentos de avaliação M0 e M2, verificou-se esta evolução e em que sentido esta se realizou. Como se pode observar, o score do instrumento EQ-5D $(\mathrm{p}<.001)$, as dimensões $\mathrm{D}(\mathrm{p}<.001)$, SSP $(\mathrm{p}=.005)$, e A $(\mathrm{p}=.005)$, do IAN, apresentaram diferenças estatisticamente significativas. 
Tabela 3 -Comparação dos três momentos de avaliação relativa à QdV, Suporte Social e Satisfação das Necessidades $(\mathrm{N}=25)$

\begin{tabular}{|c|c|c|c|c|}
\hline & \multicolumn{4}{|c|}{ Momentos de Avaliação } \\
\hline & M0 & M1 & M2 & M0 M2 \\
\hline & $\begin{array}{c}\text { Teste de } \\
\text { Friedman } \\
(\mathrm{OMa} ; \mathrm{p})\end{array}$ & $\begin{array}{l}\text { Teste de } \\
\text { Friedman } \\
(\mathrm{OM} ; \mathrm{p})\end{array}$ & $\begin{array}{c}\text { Teste de } \\
\text { Friedman } \\
(\mathrm{OM} ; \mathrm{p})\end{array}$ & $\begin{array}{l}\text { Teste de Wil- } \\
\text { coxon (p) }\end{array}$ \\
\hline \multirow[t]{2}{*}{ EQ-5D } & 1,42 & 2,00 & 2,58 & $\begin{array}{c}\text { Positivos }^{* *}=18 \\
\text { Negativos }^{* * *}= \\
1 \\
\text { Empates }^{* * * *}=6\end{array}$ \\
\hline & \multicolumn{3}{|c|}{$\mathrm{p}<.001^{\star}$} & $\mathrm{p}^{*}<.001$ \\
\hline \multirow[t]{2}{*}{ ESSS } & 1,78 & 2,04 & 2,18 & $\begin{array}{c}\text { Positivos }=15 \\
\text { Negativos }=10\end{array}$ \\
\hline & \multicolumn{3}{|c|}{$\mathrm{p}=.350$} & $\mathrm{p}=.170$ \\
\hline \multicolumn{5}{|l|}{ IAN } \\
\hline Dimensão RS & 1,90 & 1,78 & 2,32 & $\begin{array}{c}\text { Positivos }=14 \\
\text { Negativos }=8 \\
\text { Empates }=3\end{array}$ \\
\hline \multirow{2}{*}{ Dimensão D } & \multicolumn{3}{|c|}{$\mathrm{p}=.094$} & $\mathrm{p}=.107$ \\
\hline & 1,56 & 1,66 & 2,78 & $\begin{array}{l}\text { Positivos }=22 \\
\text { Negativos }=3\end{array}$ \\
\hline \multirow[t]{2}{*}{ Dimensão E } & \multicolumn{3}{|c|}{$\mathrm{p}<.001^{\star}$} & $\mathrm{p}<.001^{\star}$ \\
\hline & 2,00 & 1,72 & 2,28 & $\begin{array}{c}\text { Positivos }=15 \\
\text { Negativos }=8 \\
\text { Empates }=2\end{array}$ \\
\hline \multirow{2}{*}{$\begin{array}{l}\text { Dimensão } \\
\text { SSP }\end{array}$} & \multicolumn{3}{|c|}{$\mathrm{p}=.119$} & $\mathrm{p}=.257$ \\
\hline & 1,74 & 1,96 & 2,30 & $\begin{array}{c}\text { Positivos }=15 \\
\text { Negativos }=7 \\
\text { Empates }=3\end{array}$ \\
\hline \multirow[t]{2}{*}{ Dimensão P } & \multicolumn{3}{|c|}{$\mathrm{p}=.104$} & $\mathrm{p}=.005^{\star}$ \\
\hline & 2,04 & 1,82 & 2,14 & $\begin{array}{l}\text { Positivos }=11 \\
\text { Negativos }=14\end{array}$ \\
\hline \multirow{3}{*}{ Dimensão A } & \multicolumn{3}{|c|}{$\mathrm{p}=.508$} & $\mathrm{p}=.388$ \\
\hline & 1,62 & 1.82 & 2,56 & $\begin{array}{c}\text { Positivos }=19 \\
\text { Negativos }=4 \\
\text { Empates }=2\end{array}$ \\
\hline & \multicolumn{3}{|c|}{$\mathrm{p}<.001^{\star}$} & $\mathrm{p}=.005^{\star}$ \\
\hline
\end{tabular}

${ }^{\star} \mathrm{p}<.05 ;{ }^{* *} \mathrm{M} 2>\mathrm{M} 0 ;{ }^{* * *} \mathrm{M} 2<\mathrm{M} 0 ;{ }^{* * * *} \mathrm{M} 2=\mathrm{M} 0 ; \mathrm{aOM}=$ Ordem Média

\section{DISCUSSÃO}

Apesar da evidência ser parca relativamente a programas de intervenção na promoção do recovery da pessoa com doença mental, podemos afirmar que, de acordo com a evidência existente, este estudo vai ao encontro de outros estudos que nos indicam que as necessidades das pessoas com doença mental são abrangentes e incluem a QdV, relações interpessoais, domínio social, autoeficácia, definição e realização de objetivos e a estabilização de sintomas psiquiátricos (Gelkopf et al., 2016). Anastácio \& Furtado (2012) adiantam que estes programas devem ser orientados para o envolvimento da pessoa no processo, pressupondo uma avaliação que antecede o programa de intervenção e que devem incluir aspetos da vida que ultrapassem o recovery clínico. Este aspeto corrobora o desenho deste programa de intervenção, que pretende ir ao encontro das necessidades das pessoas, à perceção da QdV e à satisfação com o suporte social avaliadas no início do programa de intervenção (M0), sendo os participantes do estudo ativos em todo o processo, promovendo, assim, também a sua autonomia A QdV é uma dimensão importante a avaliar porque, sendo multidimensional, também nos fornece dados para a intervenção dirigida às dimensões que apresentam scores mais baixos. Tendo como referência estudos efetuados no que se refere à avaliação da QdV da pessoa com doença mental (Gameiro et al., 2010, Macedo, 2013; Figueira et al., 2010; Macedo, 2013; Vaz-Serra et al., 2010; Gameiro et al., 2008; Macedo, 2013), pareceu-nos importante que se desse um passo em frente, intervindo nesta dimensão e que os participantes alterassem positivamente, através do programa de intervenção, a perceção da $\mathrm{QdV}$, facto que se veio a observar neste estudo.

Relativamente à satisfação com o suporte social podemos afirmar que também se observou uma evolução positiva nos três momentos de avaliação, embora sem significado estatístico. Apesar disso, não podemos deixar de valorizar este resultado pois, os objetivos do programa de intervenção também preconizaram a melhoria dessa perceção, sendo estes dados corroborados por Huang, et. al. (2008), que nos indicam que o suporte social em pessoas com doença mental tem um efeito positivo na adaptação e reduz o número de internamentos e sintomas psiquiátricos. Não podemos deixar de exprimir que este estudo permitiu intervir no recovery da pessoa com doença mental, dando resposta à filosofia implícita neste processo sendo uma intervenção morosa e exigente, pois implica uma adaptação constante às dificuldades que as pessoas vão apresentando ao longo da intervenção. Torna-se evidente que é um processo em que estão envolvidas diferentes variáveis, diferentes momentos de avaliação e que os profissionais e investigadores têm de estar preparados para o imprevisto e para a reformulação das intervenções, tendo em conta os indicadores que se vão encontrando. Como limitações do estudo podemos considerar: o grupo de indivíduos estudado ter sido sujeito a uma técnica de amostragem conveniência, o que pode traduzir uma dificuldade em comparar as mudanças nas variáveis dependentes após a introdução da intervenção (Fortin, 2009); 
O IAN construído pela equipa de investigação não ser alvo de validação, uma vez que o $\mathrm{N}$ amostral foi insuficiente para a realização deste procedimento e; a ausência de grupo de controlo e consequente repartição aleatória dos participantes, o que limita a avaliação da verdadeira eficácia da implementação da intervenção.

\section{CONCLUSÕES}

Tendo em conta o objetivo do estudo, constatou-se que a implementação do programa de intervenção delineado teve um impacto positivo nos participantes do estudo, com particular relevância nas dimensões com significado estatístico: QdV (EQ-5D), Dimensão Doença e na Dimensão Ambiente, do IAN.

A análise inferencial permite-nos concluir que existe evolução positiva com grande significado na $\mathrm{QdV}$ (EQ-5D), na Dimensão Doença e na Dimensão Ambiente, do IAN.

Apesar das limitações apontadas, os resultados deste estudo devem ser interpretados tendo em conta a investigação mais alargada que ainda está a decorrer. No entanto, concluímos que o programa de intervenção referido neste estudo atendendo à $\mathrm{QdV}$, ao suporte social e à satisfação das necessidades de pessoas com doença mental, após um período de internamento, revela resultados positivos com grande potencial para a definição de modelos de intervenção personalizados, promotores do recovery nesta população.

Parece-nos que o desenho do(s) programa(s) de intervenção, quer no que diz respeito ao número de sessões, quer no que diz respeito aos objetivos propostos para cada dimensão a trabalhar com os participantes, se revelou eficaz. Neste programa em concreto, os indicadores de resultado são importantes, mas o que moveu os investigadores foram os indicadores de processo, porque foi através deles que o desenho da intervenção foi sendo reformulado.

\section{IMPLICAÇÕES PARA A PRÁTICA CLÍNICA}

Como já foi referido, e tendo em conta a pesquisa prévia que os investigadores realizaram, a evidência relativa a programas de intervenção na promoção do recovery da pessoa com doença mental ainda é muito reduzida. A intervenção nesta área, e tendo em conta as políticas nacionais e internacionais no que respeita à saúde mental e psiquiátrica, coloca-nos alguns desafios.
Desde logo, é necessário ter em conta que o acompanhamento das pessoas com doença mental não termina no internamento. Pensamos que, assim, se consegue dar resposta aos seus problemas e simultaneamente às diretrizes existentes para a assistência à pessoa com doença mental. Este estudo pretende oferecer um aporte aos profissionais para o acompanhamento da pessoa na comunidade (domicílio), após a alta hospitalar, tendo em vista a sua recuperação máxima. As variáveis aqui estudadas são importantes para incluir nos programas de intervenção, podendo ser incluídas outras que o investigador possa considerar pertinentes.

\section{Financiamento}

Este artigo foi desenvolvido no âmbito do projeto (NORTE-01-0145-FEDER-023855), cofinanciado pelo Programa Operacional Regional do Norte (NORTE 2020), através do Portugal 2020 e do Fundo Europeu de Desenvolvimento Regional (FEDER).

\section{REFERÊNCIAS BIBLIOGRÁFICAS}

Anastácio, C., Furtado, J. (2012). Reabilitação psicossocial e recovery: conceitos e influências nos serviços oferecidos pelo sistema de saúde mental. Cadernos Brasileiros de Saúde Mental, 4(9):72-83. Disponível em: http://incubadora.periodicos.ufsc.br/index.php/ cbsm/article/view/2126/2919

Cobb, S. (1976). Social support as a moderator of life stress. Psychosomatic Medicine, 38(5), 300-314. Doi: org/10.1097/00006842-197609000-00003

Ferreira, P., Ferreira, L., Pereira, L. (2013). Contribution for the Validation of the Portuguese Version of EQ-5D. Acta Médica Portuguesa, 26(6), 664-675. Disponível em: https://www.actamedicaportuguesa.com/ revista/index.php/amp/article/viewFile/1317/3908

Figueira, M., Leitão, J., \& Gameiro, J. (2010). Qualidade de vida em doentes bipolares. In M. C. Canavarro \& A. Vaz Serra (Coord.). Qualidade de vida e saúde: uma abordagem na perspectiva da Organização Mundial da Saúde (pp. 283-298). Lisboa: Fundação Calouste Gulbenkian

Fortin, M-F. (2009). Fundamentos e etapas do processo de investigação. Loures: Lusodidata. 
Gameiro, S., Carona, C., Pereira, M., Canavarro, M., Simões, M., Rijo, D., ...\& Vaz Serra, A. (2008). Sintomatologia depressiva e qualidade de vida na população geral. Psicologia, Saúde \& Doenças, 9(1), 103-112. Disponível em: http://www. scielo.mec.pt/scielo.php?script=sci_arttext\&pid $=$ S1645-00862008000100009

Gameiro, S., Carona, C., Silva, S., \& Canavarro, C. (2010). Qualidade de vida e depressão: um estudo comparativo com doentes com diagnóstico clínico de depressão major, utentes de centros de saúde e indivíduos da população geral. In M. C. Canavarro \& A. Vaz Serra (Coord.). Qualidade de vida e saúde: uma abordagem na perspectiva da Organização Mundial da Saúde (pp. 299-323). Lisboa: Fundação Calouste Gulbenkian

Gelkopf, M., Lapid, 1., Werbeloff, N., Levine, S., Telem, A., Zisman-Ilani, Y., ...\& Roe, D. (2016). A strengthsbased casemanagement servisse for people with serious mental illness in Israel:A randomized controlled trial. Psychiatry Research, 182-189. Doi: 10.1016/j. psychres.2016.04.106

Huang, CY., Sousa, VD., Tsai, CC., \& Hwang, MY. (2008). Social support and adaptation of Taiwanese adults with mental illness. Journal of Clinical Nursing, 17(13), 1795-1802. Doi: 10.1111/j.13652702.2008.02310.x

Newson, J., \& Schulz, R. (1996). Social support as a mediator in the relation between functional status and quality of life in older adults. Psychology and Aging, 11(1), 34-44. Doi: 10.1037/0882-7974.11.1.34

Macedo, E. (2013). Qualidade de vida e doença mental. (Tese de Doutoramento). Universidade de Aveiro, Portugal.

Maulik, P., Eaton, W., \& Bradshaw, C. (2009). The role of social network and support in mental health service: findings from the Baltimore ECA Study. Psychiatric Services, 60 (9), 1222 -1229. Doi: 10.1176/appi. ps.60.9.1222

Pestana, M. \& Gageiro, J. (2008). Análise de Dados para Ciências Socias - A Complementaridade do SPSS. Lisboa: Edições Síbalo
Pais Ribeiro, J.L. (2011). Escala de satisfação com o suporte social. (1. ${ }^{\mathrm{a}}$ ed.). Lisboa: Placebo Editora LDA

Slade, M. (2013). 100 ways to support recovery - A guide to mental health professionals. ( $2^{\mathrm{a}}$ ed.). London: Rethink Mental Illness. Disponível em: https:// recoverylibrary.unimelb.edu.au/__data/assets/pdf_ file/0005/1391270/100_ways_to_support_recovery. pdf

Vaz-Serra, A., Pereira, M., \& Leitão, P. (2010). Qualidade de vida em doentes esquizofrénicos. In M. C. Canavarro \& A. Vaz Serra (Coord.). Qualidade de vida e saúde: uma abordagem na perspectiva da Organização Mundial da Saúde (pp. 271-282). Lisboa: Fundação Calouste Gulbenkian

WHOQOL Group (1996). What Quality of Life?. World Health Forum, 17(4), 354-356. Disponível em: https://apps.who.int/iris/bitstream/handle/10665/54358/WHF_1996_17\%284\%29_p354356.pdf? sequence $=1$ \&isAllowed $=y$

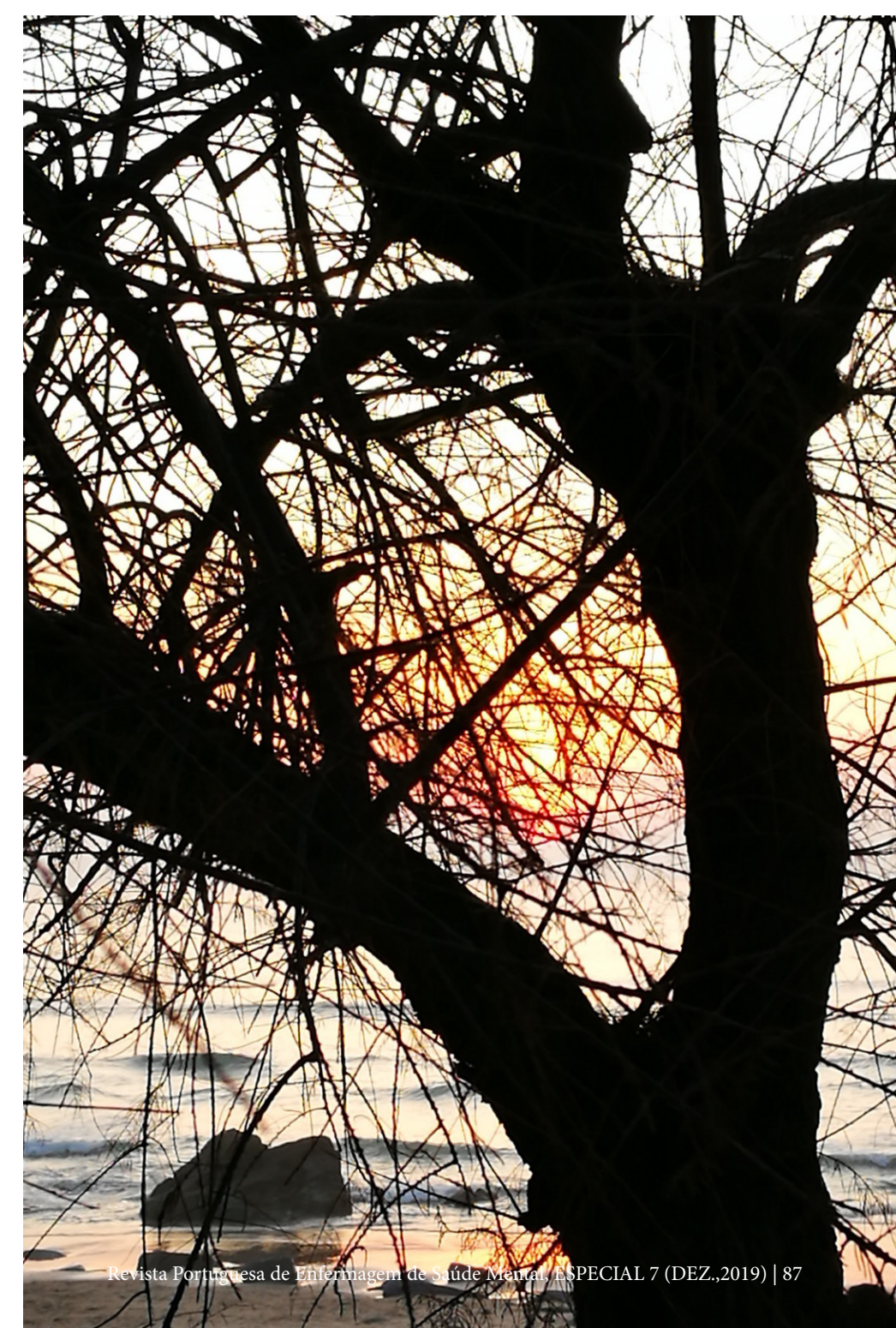

